\title{
Connecting ANITA anomalous events to a nonthermal dark matter scenario
}

\author{
Debasish Borah, ${ }^{1, *}$ Arnab Dasgupta, ${ }^{2, \dagger}$ Ujjal Kumar Dey $\odot,{ }^{3,4, \$}$ and Gaurav Tomar ${ }^{5, \S}$ \\ ${ }^{1}$ Department of Physics, Indian Institute of Technology Guwahati, Assam 781039, India \\ ${ }^{2}$ School of Liberal Arts, Seoul-Tech, Seoul 139-743, Korea \\ ${ }^{3}$ Asia Pacific Center for Theoretical Physics, Pohang 37673, Korea \\ ${ }^{4}$ Department of Physical Sciences, Indian Institute of Science Education and Research Berhampur, \\ Transit Campus, Government ITI, Berhampur 760010, Odisha, India \\ ${ }^{5}$ Department of Physics, Sogang University, Seoul 121-742, Korea
}

(Received 21 August 2019; accepted 9 April 2020; published 21 April 2020)

\begin{abstract}
The ANtarctic Impulsive Transient Antenna (ANITA) Collaboration has observed two EeV-energy, upward-going events originating from below the horizon. As no standard-model (SM) particles propagating through the Earth at such energy and exit angles can give rise to the required survival probability for the observed events, several beyond-standard-model (BSM) proposals have come up. We propose a scenario where a $Z_{2}$-odd sector is responsible for such anomalous events. The next-to-lightest $Z_{2}$-odd particle or the next-to-lightest stable particle (NLSP), created from ultrahigh-energy neutrino interactions with nucleons, can pass through the Earth and then decay into the lightest $Z_{2}$-odd particle or the lightest stable particle (LSP) and a tau lepton. The long-lived nature of the NLSP requires its coupling with the LSP to be very small, keeping the LSP out of thermal equilibrium in the early Universe. The LSP can then be a nonthermal dark matter, while the tau leptons produced from NLSP decay after passing through Earth can explain the ANITA events. We first show that a purely nonthermal dark matter scenario cannot give rise to the required event rates, while a hybrid scenario where dark matter can have thermal as well as nonthermal contribution to its relic abundance serves the purpose.
\end{abstract}

DOI: 10.1103/PhysRevD.101.075039

\section{INTRODUCTION}

Recently, the ANtarctic Impulsive Transient Antenna (ANITA) Collaboration has reported two anomalous upward-going ultrahigh-energy cosmic-ray (UHECR) air shower events corresponding to deposited energies of $0.6 \pm 0.4 \mathrm{EeV}$ and $0.56_{-0.2}^{+0.3} \mathrm{EeV}[1,2]$. These two events, which originate from well below the horizon, with angles of elevation $-27.4^{\circ} \pm 0.3^{\circ}$ and $-35.0^{\circ} \pm 0.3^{\circ}$, respectively, have $\geq 3 \sigma$ evidence for occurring due to direct upwardmoving Earth-emergent UHECR-like air showers above the Antarctic ice surface [2]. However, no particle in the standard model (SM) can survive such a passage through Earth, a distance of several thousand kilometers corresponding to the observed zenith angles for ANITA anomalous events (AAEs). Therefore, several beyond-standard-model

\footnotetext{
*dborah@iitg.ac.in

†arnabdasgupta@protonmail.ch

*ujjal@apctp.org, ujjal@iiserbpr.ac.in

\$tomar@sogang.ac.kr
}

Published by the American Physical Society under the terms of the Creative Commons Attribution 4.0 International license. Further distribution of this work must maintain attribution to the author(s) and the published article's title, journal citation, and DOI. Funded by SCOAP ${ }^{3}$.
(BSM) proposals have been put forward to explain the observed events. They include sterile neutrino mixing [3,4], heavy dark matter (DM) [5-12], inelastic boosted DM [13,14], long-lived charged particles like staus [15,16], R-parity-violating supersymmetry (SUSY) [17], SUSY sphaleron configurations [18], leptoquarks [19], and radio pulses from axion-photon conversion [20]. Additionally, in Ref. [21], the $\nu_{\tau}$ neutrino origin of AAEs is studied in compliance with Auger [22] and IceCube [23] upper limits. Note that explanations of AAEs in the SM scenario are also proposed [24,25], but they remain speculative and require experimental confirmations.

Here we propose a specific type of dark matter scenario where DM relic abundance is generated partially or fully from a nonthermal mechanism. DM contributes around $27 \%$ to the present Universe's energy density according to the latest cosmology data provided by the Planck satellite [26]. In terms of density parameter $\Omega_{\mathrm{DM}}$ and $h=$ Hubble parameter $/\left(100 \mathrm{~km} \mathrm{~s}^{-1} \mathrm{Mpc}^{-1}\right)$, the present DM abundance is conventionally reported as [26] $\Omega_{\mathrm{DM}} h^{2}=0.120 \pm 0.001$ at $68 \%$ C.L. Since none of the SM particles could provide a suitable DM candidate, several BSM proposals have come up, out of which the weakly interacting massive particle (WIMP) paradigm has been the most popular one. In this framework, a dark matter 
candidate, typically with electroweak scale mass and an interaction rate similar to electroweak interactions, can give rise to the correct dark matter relic abundance, a remarkable coincidence often referred to as the WIMP miracle. However, none of the experiments looking for direct detection of DM has found any signal yet, motivating the community to seek a paradigm shift. One such scenario that has drawn attention in the last few years is nonthermal dark matter [27]. In such a framework, DM particles have such feeble interactions with the remaining thermal bath that it never attains thermal equilibrium at any epoch in the early Universe. However, it can be produced from the decay of some heavy particles or scattering processes, popularly known as the freeze-in mechanism [27-30], leading to a new paradigm called the freeze-in (or feebly interacting) massive particle (FIMP). For a recent review of this DM paradigm, please see Ref. [31]. Typical FIMP or nonthermal DM models involve tiny couplings of DM with SM or other particles in the thermal bath and often lead to longlived particles. Such long-lived charged particles have the potential to pass through the Earth before decaying into DM and a tau lepton, which could explain the anomalous events observed by ANITA $[15,16]$.

Motivated by these, we propose a model which is an extension of the scotogenic model [32] that provides a common origin of DM and light neutrino masses. The original scotogenic model is an extension of the SM by three right-handed neutrinos and one additional scalar doublet, all of which are odd under an in-built and unbroken $Z_{2}$ symmetry. It is worth mentioning at this point that the observation of nonzero neutrino masses and large leptonic mixing [33] has also been another motivation for BSM physics for the last few decades. While the addition of singlet right-handed neutrinos to the SM content can give rise to the usual seesaw mechanism [34-36] for neutrino mass at tree level, the scotogenic framework can explain the origin of neutrino mass and dark matter in a unified manner. The $Z_{2}$-odd particles, the lightest of which is the DM candidate, give rise to light neutrino masses at one-loop level in this model. We extend this model suitably to explain the AAEs. We first consider an extension of this model to incorporate a purely nonthermal or FIMP-type DM and name it as Model I. Here, the DM is a gauge singlet right-handed neutrino, whose relic is generated purely from the nonthermal contribution, by virtue of its small couplings to SM leptons. Although correct DM properties as well as light neutrino masses can be realized in such a scenario, it fails to explain the AAEs. We then consider a hybrid setup, where the DM relic receives both thermal and nonthermal contributions. In this scenario, referred to as Model II hereafter, DM is the lightest neutral component of the $Z_{2}$-odd scalar doublet. Due to electroweak gauge interactions, the DM in this model cannot be of purely nonthermal origin. However, it can receive a nonthermal contribution from the decay of heavier particles after it freezes out, similar to the recent Refs. [37,38]. In such scenarios, the thermally underabundant DM can satisfy the correct relic abundance criteria due to the late nonthermal contributions. We show how the correct DM and neutrino phenomenology can be obtained in this hybrid model, along with the explanation for the AAEs.

This paper is organized as follows: In Secs. II A and II B, we discuss the two models along with the corresponding DM phenomenology. In Sec. III, we discuss the origin of ANITA anomalous events in both the models. We briefly comment upon different ways to probe our models in Sec. IV, and finally conclude in Sec. V.

\section{MODEL}

\section{A. Model I: Pure freeze-in}

As mentioned earlier, we first discuss a model where the DM relic is generated purely from the freeze-in mechanism. The particle content of Model I is shown in Table I. There are five different types of BSM fields, all of which are odd under an in-built and unbroken $Z_{2}$ symmetry. We need three copies of the singlet right-handed neutrino $N$ in order to account for nonthermal DM and light neutrino masses simultaneously. The lightest of the right-handed neutrinos, $N_{1}$, is the DM candidate having tiny Yukawa couplings with leptons, while the heavier right-handed neutrinos generate the light neutrino masses at radiative level. While $\Phi_{2}$ and $N$ are the same as those in the minimal scotogenic model and are sufficient to realize $N_{1}$ as freeze-in DM and generate light neutrino masses and mixing, the other three types of particles, $E_{L, R}, \psi_{1}$, and $\psi_{2}$, are required in order to explain the AAEs. It is worth mentioning that due to very small Yukawa couplings associated with one of the right-handed neutrinos $N_{1}$, the lightest neutrino mass becomes vanishingly small, predicting a hierarchical light neutrino mass spectrum.

The relevant part of the Yukawa Lagrangian is given by

$$
\begin{aligned}
\mathcal{L}_{Y} \supset & Y_{1} \bar{L} \Phi_{1} e_{R}+Y_{2} \bar{L} \Phi_{2} E_{R}+Y_{2}^{\prime} L^{T} \widetilde{\Phi_{2}} E_{L}+Y_{3} \bar{L} \widetilde{\Phi_{2}} N \\
& +Y_{4} \overline{u_{R}} \psi_{1}^{\dagger} E_{L}+Y_{5} \overline{d_{R}} \psi_{2}^{\dagger} E_{L} \\
& +Y_{4}^{\prime} u_{R} \psi_{1} E_{R}+Y_{5}^{\prime} d_{R} \psi_{2} E_{R}+Y_{6} d_{R} \psi_{1} N .
\end{aligned}
$$

The scalar potential is

TABLE I. Field content and transformation properties under the symmetry of the model.

\begin{tabular}{lccccccccccc}
\hline \hline Fields & $Q$ & $u_{R}$ & $d_{R}$ & $L$ & $e_{R}$ & $\Phi_{1}$ & $\Phi_{2}$ & $E_{L, R}$ & $N$ & $\psi_{1}$ & $\psi_{2}$ \\
\hline$S U(3)$ & 3 & 3 & 3 & 1 & 1 & 1 & 1 & 1 & 1 & 3 & 3 \\
$S U(2)$ & 2 & 1 & 1 & 2 & 1 & 2 & 2 & 1 & 1 & 1 & 1 \\
$U(1)_{Y}$ & $\frac{1}{6}$ & $\frac{2}{3}$ & $-\frac{1}{3}$ & $-\frac{1}{2}$ & -1 & $\frac{1}{2}$ & $\frac{1}{2}$ & \pm 1 & 0 & $\frac{1}{3}$ & $\frac{4}{3}$ \\
$Z_{2}$ & 1 & 1 & 1 & 1 & 1 & 1 & -1 & -1 & -1 & -1 & -1 \\
\hline \hline
\end{tabular}




$$
\begin{aligned}
V\left(\Phi_{1}, \Phi_{2}, \psi_{1}, \psi_{2}\right)= & \mu_{1}^{2}\left|\Phi_{1}\right|^{2}+\mu_{2}^{2}\left|\Phi_{2}\right|^{2}+\frac{\lambda_{1}}{2}\left|\Phi_{1}\right|^{4}+\frac{\lambda_{2}}{2}\left|\Phi_{2}\right|^{4}+\lambda_{3}\left|\Phi_{1}\right|^{2}\left|\Phi_{2}\right|^{2} \\
& +\lambda_{4}\left|\Phi_{1}^{\dagger} \Phi_{2}\right|^{2}+\left\{\frac{\lambda_{5}}{2}\left(\Phi_{1}^{\dagger} \Phi_{2}\right)^{2}+\text { H.c. }\right\}+\mu_{3}^{2}\left|\psi_{1}\right|^{2}+\mu_{4}^{2}\left|\psi_{2}\right|^{2} \\
& +\left|\Phi_{1}\right|^{2}\left(\lambda_{6}\left|\psi_{1}\right|^{2}+\lambda_{7}\left|\psi_{2}\right|^{2}\right)+\left|\Phi_{2}\right|^{2}\left(\lambda_{8}\left|\psi_{1}\right|^{2}+\lambda_{9}\left|\psi_{2}\right|^{2}\right) \\
& +\left\{\frac{\lambda_{10}}{2}\left(\Phi_{1}^{\dagger}\right)^{2}\left(\psi_{1}^{\dagger} \psi_{2}\right)+\text { H.c. }\right\}+\left\{\frac{\lambda_{11}}{2}\left(\Phi_{2}^{\dagger}\right)^{2}\left(\psi_{1}^{\dagger} \psi_{2}\right)+\text { H.c. }\right\}+\lambda_{12}\left|\psi_{1}\right|^{4}+\lambda_{13}\left|\psi_{2}\right|^{4} .
\end{aligned}
$$

As mentioned, the lightest right-handed neutrino $N_{1}$ is the dark matter candidate in this model, and being gauge singlet, it can be prevented from being produced thermally in the early Universe, if the corresponding Yukawa couplings are very small. We consider such a possibility where $N_{1}$ is produced nonthermally from the decay of $\Phi_{2}$. The decay of $\Phi_{2}$ can occur either in equilibrium or after its freeze-out; the latter is similar to the super-WIMP formalism [39]. It should be noted that all the components of $\Phi_{2}$ can contribute to the nonthermal production of $N_{1}$ : namely, $\Phi_{2}^{ \pm} \rightarrow N_{1} l^{ \pm}, \Phi_{2}^{0} \rightarrow N_{1} \nu, l \equiv e, \mu, \tau$. Since the $Z_{2}$ symmetry remains unbroken, the neutral components of $\Phi_{2}$ do not acquire any nonzero vacuum expectation value (VEV), which can be ensured by choosing $\mu_{2}^{2}>0$. We parametrize the two scalar doublets $\Phi_{1,2}$ as

$$
\Phi_{1}=\left(\begin{array}{c}
0 \\
\frac{1}{\sqrt{2}}(v+h)
\end{array}\right), \quad \Phi_{2}=\left(\begin{array}{c}
\Phi_{2}^{+} \\
\frac{1}{\sqrt{2}}\left(\Phi_{2}^{R}+i \Phi_{2}^{I}\right)
\end{array}\right),
$$

where $\Phi_{1}$ is identified with the SM Higgs doublet, whose neutral component acquires a VEV denoted by $v$, responsible for electroweak symmetry breaking (EWSB). As follows from the scalar potential, after EWSB, the physical scalars (originating from the two scalar doublets) have the following masses:

$$
\begin{gathered}
m_{h}^{2}=\frac{1}{2} \lambda_{1} v^{2}, \\
\left(M_{\Phi_{2}}^{R}\right)^{2}=\mu_{2}^{2}+\frac{1}{2}\left(\lambda_{3}+\lambda_{4}+\lambda_{5}\right) v^{2}, \\
\left(M_{\Phi_{2}}^{I}\right)^{2}=\mu_{2}^{2}+\frac{1}{2}\left(\lambda_{3}+\lambda_{4}-\lambda_{5}\right) v^{2}, \\
\left(M_{\Phi_{2}}^{ \pm}\right)^{2}=\mu_{2}^{2}+\frac{1}{2} \lambda_{3} v^{2} .
\end{gathered}
$$

The relevant parameters as well as the physical masses of different fields are listed in Table II. The other parameters which do not find mention in this table can be chosen as per phenomenological requirements, and we do not list them in this table as they do not affect our numerical calculations.

In order to compute the abundance of $N_{1}$, we first write down two Boltzmann equations corresponding to the evolution of $\Phi_{2}^{ \pm}$as well as $N_{1}$. They can be written in terms of their comoving number densities $(Y=n / s$, $n \equiv$ number density, $s \equiv$ entropy density) as

$$
\begin{gathered}
\frac{d Y_{\Phi_{2}^{ \pm}}}{d z}=-\frac{\langle\sigma v\rangle s}{H z}\left(Y_{\Phi_{2}^{ \pm}}^{2}-\left(Y_{\Phi_{2}^{ \pm}}^{\mathrm{eq}}\right)^{2}\right)-\frac{\left\langle\Gamma_{\Phi_{2}^{ \pm}}\right\rangle}{H z} Y_{\Phi_{2}^{ \pm}}, \\
\frac{d Y_{N_{1}}}{d z}=\frac{\left\langle\Gamma_{\Phi_{2}^{ \pm}}\right\rangle}{H z} Y_{\Phi_{2}^{ \pm}},
\end{gathered}
$$

with $z=M_{\Phi_{2}^{ \pm}} / T$ and a thermally averaged decay width of $\Phi_{2}^{ \pm}$,

$$
\begin{aligned}
\left\langle\Gamma_{\Phi_{2}^{ \pm}}\right\rangle= & \frac{Y_{3}^{2}}{8 \pi} M_{\Phi_{2}^{ \pm}}\left(1-\left(\frac{M_{N_{1}}+m_{\tau}}{M_{\Phi_{2}^{ \pm}}}\right)^{2}\right)^{3 / 2} \\
& \times\left(1-\left(\frac{M_{N_{1}}-m_{\tau}}{M_{\Phi_{2}^{ \pm}}}\right)^{2}\right)^{1 / 2} \frac{K_{1}\left(M_{\Phi_{2}^{ \pm}} / T\right)}{K_{2}\left(M_{\Phi_{2}^{ \pm}} T\right)} \\
= & \Gamma_{\Phi_{2}^{ \pm}} \frac{K_{1}\left(M_{\Phi_{2}^{ \pm}} / T\right)}{K_{2}\left(M_{\Phi_{2}^{ \pm}} / T\right)}
\end{aligned}
$$

where $K_{i}$ 's are the modified Bessel functions. Now, for most of the parameter space of the Yukawa $Y_{3}$, the freeze-in

TABLE II. Numerical values of different relevant parameters used for Model I.

\begin{tabular}{lc}
\hline \hline Parameters & Benchmark \\
\hline$\mu_{2}$ & $1 \mathrm{TeV}$ \\
$\mu_{3}$ & $6 \mathrm{TeV}$ \\
$\mu_{4}$ & $6 \mathrm{TeV}$ \\
$Y_{3}$ & $2.92 \times 10^{-10}$ \\
$Y_{2}, Y_{4}, Y_{5}$ & 3.5 \\
$M_{\Phi_{2}}^{ \pm}$ & $1 \mathrm{TeV}$ \\
$M_{\Phi_{2}}^{I}$ & $1.045 \mathrm{TeV}$ \\
$M_{\Phi_{2}}^{R}$ & $1.073 \mathrm{TeV}$ \\
$\lambda_{1}$ & 0.255 \\
$\lambda_{2}$ & 0.1 \\
$\lambda_{3}$ & 0 \\
$\lambda_{4}$ & 4.0 \\
$\lambda_{5}$ & 1.0 \\
$M_{N_{1}}$ & $30 \mathrm{MeV}$ \\
$M_{E}$ & $7 \mathrm{TeV}$ \\
\hline \hline
\end{tabular}




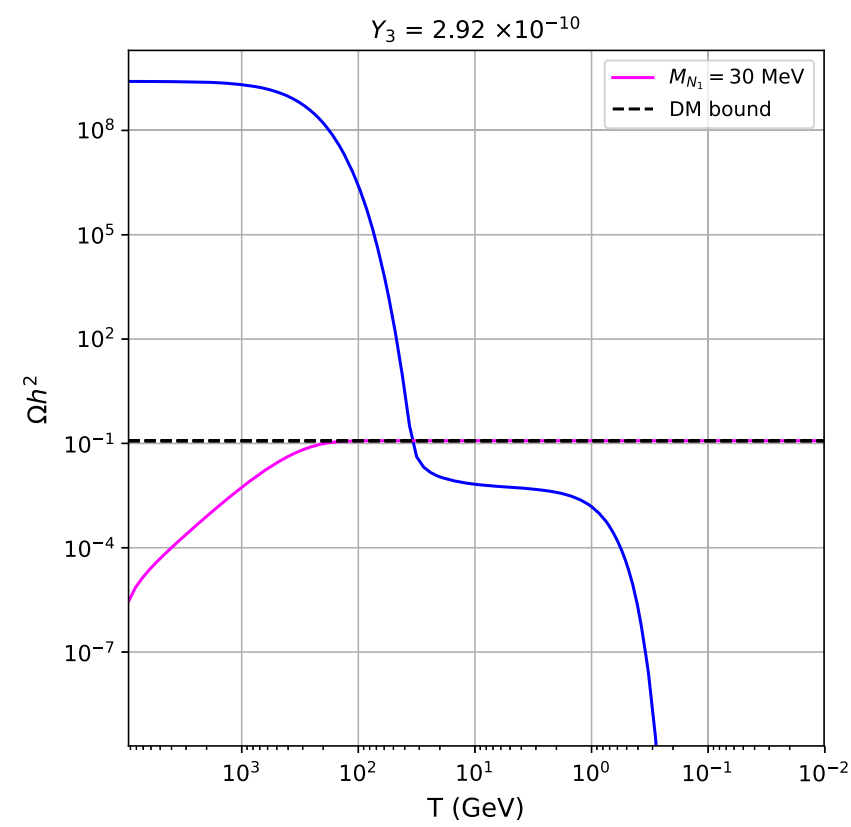

FIG. 1. Evolution of comoving number densities for nonthermal DM $\left(N_{1}\right)$ (solid magneta line) and lightest component of $\Phi_{2}$ (solid blue line). The dashed horizontal line corresponds to the Planck limit on the DM relic.

process happens while $\Phi_{2}^{ \pm}$is still in equilibrium. So, we can safely integrate Eq. (6). The solution of Eq. (6) is given as

$$
\begin{aligned}
Y_{N_{1}} & =\int_{0.1}^{\infty} z^{3} K_{1}(z) d z\left[\frac{\Gamma_{\Phi_{2}^{ \pm}}}{16 \pi^{4} H\left(M_{\Phi_{2}^{ \pm}}\right)} \frac{45}{g_{*}}\right] \\
& \simeq 4.71239\left[\frac{\Gamma_{\Phi_{2}^{ \pm}}}{16 \pi^{4} H\left(M_{\Phi_{2}^{ \pm}}\right)} \frac{45}{g_{*}}\right],
\end{aligned}
$$

and the relic is given as

$$
\Omega_{\mathrm{DM}} h^{2}=M_{N_{1}} \frac{\rho_{0}}{\rho_{c}} Y_{N_{1}} \simeq 2.345 \times 10^{25}\left(\frac{Y_{3}^{2} M_{N_{1}}}{g_{*}\left(M_{\Phi_{2}^{ \pm}}\right) M_{\Phi_{2}^{ \pm}}}\right) .
$$

For a chosen benchmark point, the temperature evolution of comoving number densities for DM and its mother particle are shown as a function of temperature in Fig. 1. We choose the benchmark point as $M_{N_{1}}=30 \mathrm{MeV}$, $M_{\Phi_{2}^{ \pm}}=1 \mathrm{TeV}, Y_{3}=2.92 \times 10^{-10}$ and find that the correct relic density is satisfied. It can be clearly seen from Fig. 1 that the number density of DM that is $N_{1}$ increases as temperature cools down due to decay of different components of $\Phi_{2}$. The equilibrium contribution of $\Phi_{2}^{ \pm}$decay dominates over its post-freeze-out contribution to the abundance of $N_{1}$ for the chosen benchmark. While $N_{1}$ receives nonthermal contribution from all the components of $\Phi_{2}$, the coannihilations among different components of $\Phi_{2}$ have been incorporated, following Ref. [40]. While the

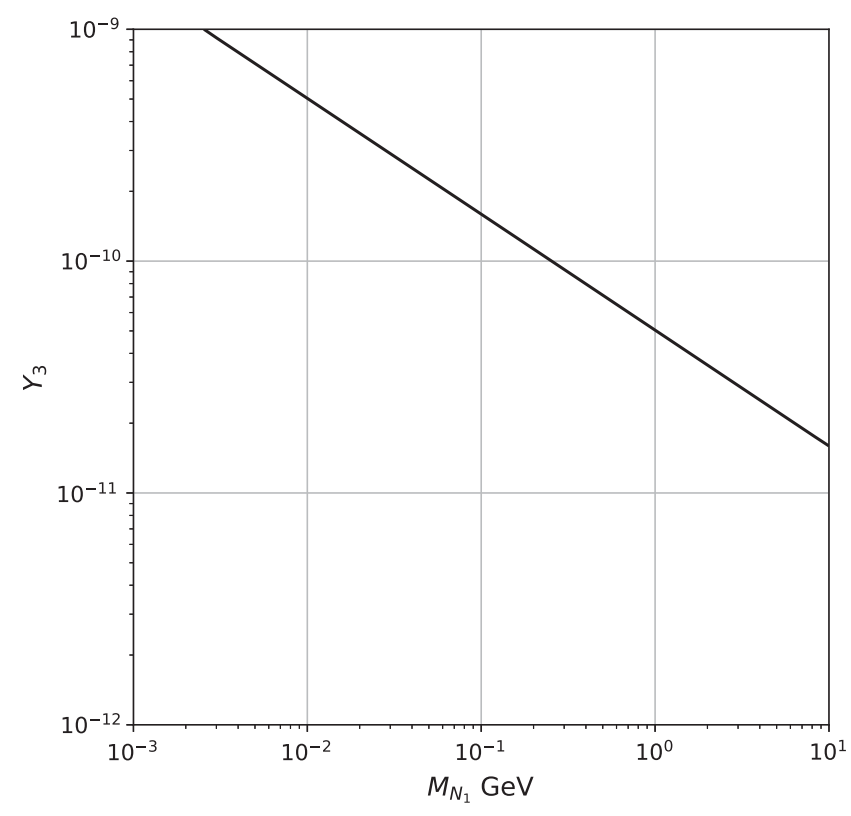

FIG. 2. Parameter space in the $Y_{3}-M_{N_{1}}$ plane which gives rise to the correct DM relic.

plot shown in Fig. 1 corresponds to a particular benchmark point of DM mass and Yukawa coupling, we show the allowed parameter space in terms of these two parameters in Fig. 2 which gives rise to the correct DM relic. While $Y_{3}$ and $M_{N_{1}}$ are being varied to generate this plot, the other parameters are kept fixed at values mentioned in Table II. The observed correlation between $Y_{3}$ and $M_{N_{1}}$ from this plot agrees with the approximate analytical expression for DM relic density derived above. Since freeze-in abundance increases with an increase in DM coupling [27], the corresponding DM mass needs to be smaller in order to be in agreement with the observed abundance. It should also be noted that the requirement of such small Yukawa coupling of DM $N_{1}$ for its nonthermal nature effectively decouples one of the singlet neutrinos from the light neutrino mass-generation mechanism at one-loop level. The other two heavy singlet neutrinos $N_{2,3}$ can, however, have order-1 couplings with leptons and can generate the required light neutrino mass. Therefore, the model predicts vanishingly small lightest neutrino mass. Although the DM relic is satisfied for this model, it cannot explain the AAEs, as we discuss in the upcoming section. Before that, we outline Model II, which satisfies all relevant DM constraints apart from explaining the AAEs.

\section{B. Model II: Freeze-out + freeze-in}

In this subsection, we discuss our Model II, where the DM relic is generated from a hybrid setup consisting of both freeze-out and freeze-in contributions. The particle content of this model is shown in Table III. There exist six different types of fields, apart from the SM fields, all of 
which are odd under an in-built $Z_{2}$ symmetry. The SM fields, on the other hand, are even under $Z_{2}$ symmetry. In order to generate correct neutrino mass, we require at least two copies of right-handed singlet neutrinos $N$. While the minimal scotogenic model has only two different types of $Z_{2}$-odd particles, the roles of the other four types of $Z_{2}$-odd particles in this model will become clear when we discuss the explanation of the AAEs in the upcoming section.

The relevant part of the Yukawa Lagrangian is given by
TABLE III. Field content and transformation properties under the symmetry of the model.

\begin{tabular}{lcccccccccccc}
\hline \hline Fields & $Q$ & $u_{R}$ & $d_{R}$ & $L$ & $e_{R}$ & $\Phi_{1}$ & $\Phi_{2}$ & $\Phi_{3}$ & $E_{L, R}$ & $N$ & $U_{L, R}$ & $D_{L, R}$ \\
\hline$S U(3)$ & 3 & 3 & 3 & 1 & 1 & 1 & 1 & 1 & 1 & 1 & 3 & 3 \\
$S U(2)$ & 2 & 1 & 1 & 2 & 1 & 2 & 2 & 2 & 1 & 1 & 1 & 1 \\
$U(1)_{Y}$ & $\frac{1}{6}$ & $\frac{2}{3}$ & $-\frac{1}{3}$ & $-\frac{1}{2}$ & -1 & $\frac{1}{2}$ & $\frac{1}{2}$ & $\frac{1}{2}$ & \pm 1 & 0 & $\pm \frac{2}{3}$ & $\mp \frac{1}{3}$ \\
$Z_{2}$ & 1 & 1 & 1 & 1 & 1 & 1 & -1 & -1 & -1 & -1 & -1 & -1 \\
\hline \hline
\end{tabular}

$$
\begin{aligned}
\mathcal{L}_{Y} \supset & Y_{1} \bar{L} \Phi_{1} e_{R}+Y_{2} \bar{L} \Phi_{2} E_{R}+Y_{3} \bar{L} \widetilde{\Phi_{2}} N+Y_{4} \bar{Q} \widetilde{\Phi_{2}} U_{R}+Y_{5} \bar{Q} \Phi_{2} D_{R} \\
& +Y_{2}^{\prime} \bar{L} \Phi_{3} E_{R}+\widetilde{Y_{3}^{\prime}} \bar{L} \widetilde{\Phi_{3}} N+Y_{4}^{\prime} \overline{\Phi_{3}} U_{R}+Y_{5}^{\prime} \overline{Q^{\prime}} \Phi_{3} D_{R} \\
& +Y_{2}^{\prime \prime} L^{T} \widetilde{\Phi_{2}} E_{L}++Y_{3}^{\prime \prime} L^{T} \widetilde{\Phi_{3}} E_{L} .
\end{aligned}
$$

The scalar potential is

$$
\begin{aligned}
V\left(\Phi_{1}, \Phi_{2}, \Phi_{3}\right)= & \mu_{1}^{2}\left|\Phi_{1}\right|^{2}+\mu_{2}^{2}\left|\Phi_{2}\right|^{2}+\mu_{3}^{2}\left|\Phi_{3}\right|^{2}+\frac{\lambda_{1}}{2}\left|\Phi_{1}\right|^{4}+\frac{\lambda_{2}}{2}\left|\Phi_{2}\right|^{4}+\frac{\lambda_{3}}{2}\left|\Phi_{3}\right|^{4} \\
& +\lambda_{4}\left|\Phi_{1}\right|^{2}\left|\Phi_{2}\right|^{2}+\lambda_{5}\left|\Phi_{1}^{\dagger} \Phi_{2}\right|^{2}+\left\{\frac{\lambda_{6}}{2}\left(\Phi_{1}^{\dagger} \Phi_{2}\right)^{2}+\text { H.c. }\right\} \\
& +\lambda_{4}^{\prime}\left|\Phi_{1}\right|^{2}\left|\Phi_{3}\right|^{2}+\lambda_{5}^{\prime}\left|\Phi_{1}^{\dagger} \Phi_{3}\right|^{2}+\left\{\frac{\lambda_{6}^{\prime}}{2}\left(\Phi_{1}^{\dagger} \Phi_{3}\right)^{2}+\text { H.c. }\right\} \\
& +\left\{\mu_{23}^{2} \Phi_{2}^{\dagger} \Phi_{3}+\text { H.c. }\right\}+\lambda_{7}\left|\Phi_{2}\right|^{2}\left|\Phi_{3}\right|^{2}+\lambda_{8}\left|\Phi_{2}^{\dagger} \Phi_{3}\right|^{2} \\
& +\left\{\lambda_{7}^{\prime}\left|\Phi_{1}\right|^{2} \Phi_{2}^{\dagger} \Phi_{3}+\lambda_{8}^{\prime} \Phi_{2}^{\dagger} \Phi_{3}\left|\Phi_{3}\right|^{2}+\lambda_{9}^{\prime} \Phi_{3}^{\dagger} \Phi_{2}\left|\Phi_{2}\right|^{2}+\text { H.c. }\right\} \\
& +\left\{\frac{\lambda_{9}}{2}\left(\Phi_{2}^{\dagger} \Phi_{3}\right)^{2}+\text { H.c. }\right\} .
\end{aligned}
$$

We assume the neutral component $\Phi_{3}^{0}$ of $\Phi_{3}$ to be the lightest $Z_{2}$-odd particle, and hence the DM candidate, while $E$ is the NLSP. Due to the long-lived singlet fermion $E$ which decays at late times to dark matter and a tau lepton, we can have a nonthermal contribution to the freeze-out abundance of DM. The physical masses of the components of scalar doublets $\Phi_{2}, \Phi_{3}$ after EWSB can be found in a way similar to Model I. However, due to the presence of these two $Z_{2}$-odd scalar doublets, there can be mixing between them as well. For simplicity, we ignore such mixing, which amounts to tuning the respective bilinear and quartic couplings like $\mu_{23}, \lambda_{7}, \lambda_{8}, \lambda_{9}$ in the scalar potential [Eq. (11)].

The coupled Boltzmann equations can be written as

$$
\begin{aligned}
\frac{d n_{\Phi_{3}^{0}}}{d t}+3 H n_{\Phi_{3}^{0}}= & -\langle\sigma v\rangle_{\Phi_{3}^{0} \Phi_{3}^{0} \rightarrow \operatorname{SM~SM}}\left(n_{\Phi_{3}^{0}}^{2}-\left(n_{\Phi_{3}^{0}}^{\mathrm{eq}}\right)^{2}\right) \\
& +N_{\Phi_{3}^{0} \Gamma_{E} n_{E}}, \\
\frac{d n_{E}}{d t}+3 H n_{E}=- & \langle\sigma v\rangle_{E E \rightarrow \operatorname{SMSM}}\left(n_{E}^{2}-\left(n_{E}^{\mathrm{eq}}\right)^{2}\right)-\Gamma_{E} n_{E} .
\end{aligned}
$$

Here, $N_{\Phi_{3}^{0}}$ is the average number of DM particles produced from a single decay of $E$, which is 1 in this case; $\Gamma_{E}$ is the decay width of $E$; and $H$ is the Hubble parameter. Assuming that the singlet fermion $E$ does not contribute dominantly to the total energy budget, we can take the comoving entropy density $\left(g_{\star s}\right)$ and the comoving energy density $\left(g_{\star}\right)$ to be approximately constant. Further, we assume that almost all of $E$ decays during the radiationdominated epoch, which is satisfied for the benchmark values of Yukawa couplings we use here. Also, if we assume $E$ decays only after its thermal freeze-out, the first term on the right-hand side of Eq. (13) can be ignored, ${ }^{1}$ and one can analytically solve the Boltzmann equation for $n_{E}$ above. Writing the above equations in terms of $Y_{\Phi_{3}^{0}}=\frac{n_{\Phi_{3}^{0}}}{s}$, $Y_{E}=\frac{n_{E}}{s}$ with $s=\frac{2 \pi^{2}}{45} g_{* s} T^{3}$ being the entropy density and changing the variable from time $t$ to $z=M_{\Phi_{3}^{0}} / T$, where $M_{\Phi_{3}^{0}}$ is the mass of DM $\Phi_{3}^{0}$, we get

\footnotetext{
${ }^{1}$ Note that the first term on the right-hand side of Eq. (13) can also be ignored if $E$ decays while in thermal equilibrium $n_{E}=n_{E}^{\text {eq }}$; we consider the general case in our numerical calculations.
} 
TABLE IV. Numerical values of different relevant parameters used for Model II.

\begin{tabular}{lcc}
\hline \hline Parameters & First benchmark & Second benchmark \\
\hline$\mu_{2}$ & $400 \mathrm{GeV}$ & $300 \mathrm{GeV}$ \\
$Y_{2}^{\prime}, Y_{2}^{\prime \prime}$ & $1.1 \times 10^{-9}$ & $4.9 \times 10^{-10}$ \\
$Y_{2}, Y_{4}, Y_{5}$ & 0.96 & 1.27 \\
$\lambda_{1}$ & 0.255 & 0.255 \\
$\lambda_{2}$ & 0.1 & 0.1 \\
$\lambda_{3}$ & 0.81 & 0.81 \\
$\lambda_{4}$ & -0.3 & -0.3 \\
$\lambda_{5}$ & $1 \times 10^{-8}$ & $1 \times 10^{-8}$ \\
$M_{\Phi_{2}^{ \pm}}$ & $1.1 \mathrm{TeV}$ & $1.1 \mathrm{TeV}$ \\
$M_{\Phi_{3}^{0}}$ & $416.2 \mathrm{GeV}$ & $321.4 \mathrm{GeV}$ \\
$M_{\Phi_{3}^{ \pm}}$ & $427.6 \mathrm{GeV}$ & $335.9 \mathrm{GeV}$ \\
$M_{U}$ & $5 \mathrm{TeV}$ & $5 \mathrm{TeV}$ \\
$M_{D}$ & $5 \mathrm{TeV}$ & $5 \mathrm{TeV}$ \\
$M_{E}$ & $1 \mathrm{TeV}$ & $1 \mathrm{TeV}$ \\
$M_{N}$ & $5 \mathrm{TeV}$ & $5 \mathrm{TeV}$ \\
\hline \hline
\end{tabular}

$$
\begin{gathered}
\frac{d Y_{\Phi_{3}^{0}}}{d z}=-\frac{\langle\sigma v\rangle s}{H z}\left(Y_{\Phi_{3}^{0}}^{2}-\left(Y_{\Phi_{3}^{0}}^{\mathrm{eq}}\right)^{2}\right)+\frac{N_{\Phi_{3}^{0}} \Gamma_{N}}{H z} Y_{E}, \\
\frac{d Y_{E}}{d z}=-\frac{\Gamma_{E}}{H z} Y_{E} .
\end{gathered}
$$

The equation for $Y_{E}$ can be solved analytically, giving

$$
Y_{E}(z)=Y_{E}\left(z_{F}\right) \exp \left(-\frac{r}{2}\left(z^{2}-z_{F}^{2}\right)\right) .
$$

Here, $z_{F}=M_{\Phi_{3}^{0}} / T_{F}$ is the point of freeze-out and usually takes a value of $\mathcal{O}(20)$. Also, $r=\frac{\Gamma_{E}}{H z^{2}}=\frac{\Gamma_{E} M_{\mathrm{Pl}}}{\pi M_{\Phi_{3}^{0}}^{0}} \sqrt{90 / g_{\star}}$

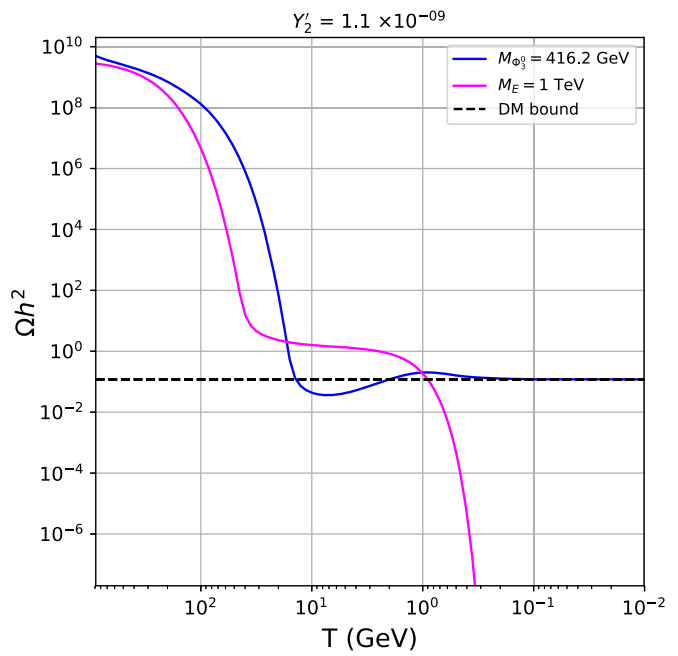

depends on the decay width of the mother particle $E$. The value of $Y_{E}\left(z_{0}\right)$ (where $z_{0}$ corresponds to the epoch after the freeze-out of $E$ from where we start integrating the differential equation for DM), however, depends on the initial abundance of $E$ and can be found by calculating its freeze-out abundance. Using this solution for $Y_{E}$, the equation for $Y_{\Phi_{3}^{0}}$ can be rewritten as

$$
\begin{aligned}
\frac{d Y_{\Phi_{3}^{0}}}{d z}= & -\frac{\langle\sigma v\rangle s}{H z}\left(Y_{\Phi_{3}^{0}}^{2}-\left(Y_{\Phi_{3}^{0}}^{\mathrm{eq}}\right)^{2}\right) \\
& +N_{\Phi_{3}^{0} r} r Y_{E}\left(z_{0}\right) \exp \left(-\frac{r}{2}\left(z^{2}-z_{0}^{2}\right)\right) .
\end{aligned}
$$

The decay width of fermion $E$ (assuming $Y_{3}^{\prime}=Y_{3}^{\prime \prime}=$ $Y_{2}^{\prime} / 2$ ), ignoring the mass of leptons, is given by

$$
\Gamma_{E}=\frac{Y_{2}^{\prime 2}}{16 \pi} M_{E}\left(1-\frac{M_{\Phi_{3}^{0}}^{2}}{M_{E}^{2}}\right)^{2}
$$

where $M_{E}$ is the mass of fermion $E$, and $Y_{2}^{\prime}$ is its coupling with the tau lepton and DM. The lifetime requirement of $E$ from an ANITA anomaly point of view is [15]

$$
\tau_{E}=\frac{1}{\Gamma_{E}} 10\left(\frac{M_{E}}{500 \mathrm{GeV}}\right) \mathrm{ns} .
$$

For $M_{E} \sim 5 \mathrm{TeV}$, the required lifetime of $100 \mathrm{~ns}$ can be obtained for $Y_{2}^{\prime} \approx 10^{-10}-10^{-9}$, a typical coupling for nonthermal dark matter. It should be noted that $E$ is produced by interactions of high-energy neutrinos with nucleons in a process mediated by the charged component of $\Phi_{2}$. The production cross section of $E$ in this process can be made large by suitable tuning of the corresponding Yukawa couplings $Y_{2}, Y_{4}, Y_{5}, Y_{2}^{\prime \prime}$. By keeping $\Phi_{2}$ heavier than

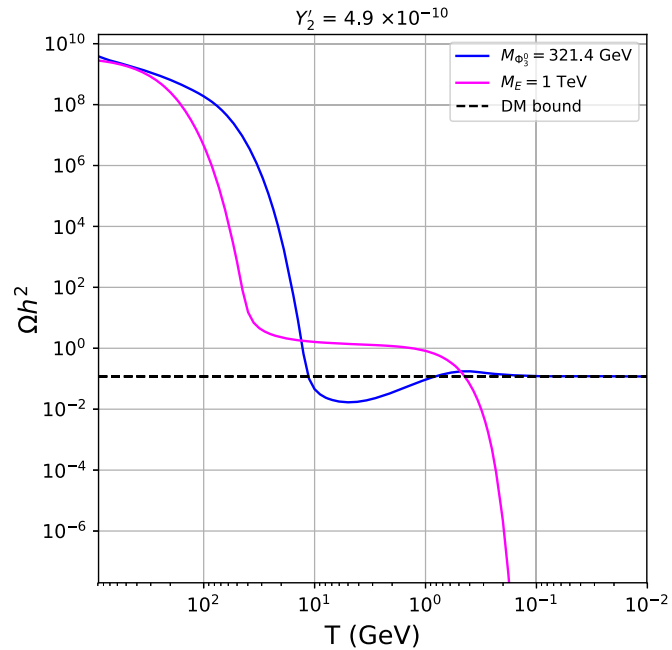

FIG. 3. Evolution of comoving number densities for DM $\left(\Phi_{3}^{0}\right)$ (solid blue line) and mother particle $E$ (solid magneta line) giving a nonthermal contribution to the DM relic at late epochs. The dashed horizontal line corresponds to the Planck limit on the DM relic. The left- and right-panel plots correspond to two different benchmark choices for Model II given in Table IV. 
$E$, in order to forbid the latter's decay into the former, we can have long-lived $E$ which decays after traveling through Earth into dark matter and tau leptons. We will discuss the details of AAEs in this model in the upcoming section.

In order to show the DM relic density results, we choose two benchmark points, the details of which are given in Table IV. For the chosen benchmark points, we then solve the coupled Boltzmann equations for $\Phi_{3}^{0}, E$ and show their comoving number densities as a function of temperature in Fig. 3. As can be seen from the plots, the comoving number densities of both $\Phi_{3}^{0}$ and $E$ decrease as the temperature falls below their respective masses, owing to the Boltzmann suppression for nonrelativistic species. At some epochs, the individual rate of interactions of $E$ as well as $\Phi_{3}^{0}$ with the SM particles freezes out, giving rise to the plateau-shaped regions. However, since $E$ is unstable and decays into $\Phi_{3}^{0}$, the comoving number density of $E$ drops very fast at some epoch due to its decay with a corresponding rise in the number density of $\Phi_{3}^{0}$. In the absence of $E$ decay, the freeze-out abundance of $\Phi_{3}^{0}$ remains underabundant compared to the Planck bound. The mass of $\Phi_{3}^{0}$ in both the benchmark points given in Table IV falls in the mass regime $80 \mathrm{GeV} \leq M_{\Phi_{3}^{0}} \leq 500 \mathrm{GeV}$, where scalar doublet DM fails to give rise to the correct thermal relic, as is well known from earlier studies on $Z_{2}$-odd scalar doublet dark matter. The role of nonthermal contribution in filling this deficit was discussed in earlier work [37].

\section{EXPLANATION OF ANITA EVENTS}

The estimation of the number of AAEs can be facilitated from the evaluation of survival probability $\epsilon$, which basically represents the fraction of incoming ultrahighenergy neutrino flux that ultimately gives rise to $\tau$ leptons near the Earth's surface. The total number of events can be estimated as

$$
\mathcal{N}=A_{\text {eff }} T \Delta \Omega \int_{E_{i}}^{E_{f}} d E_{\nu} \Phi\left(E_{\nu}\right) \epsilon\left(E_{\nu}\right),
$$

where the effective area $A_{\text {eff }} \approx 4 \mathrm{~km}^{2} ; T$ is the exposure time, which we take $\approx 25$ days by combining the exposures of ANITA-I and ANITA-III; and $\Delta \Omega$ is the acceptance angle, which, corresponding to isotropic and anisotropic neutrino flux sources, is given as

$\Delta \Omega \approx\left\{\begin{array}{l}2 \pi \text { sr for isotropic case, } \\ 2 \pi\left(1-\cos \delta_{\theta}\right) \approx 0.0022 \text { sr for anisotropic case, }\end{array}\right.$

where $\delta_{\theta} \approx 1.5^{\circ}$ is the angular uncertainty relative to the parent neutrino direction. Note that we have not considered the exposure time for ANITA-II, as it was not sensitive to such events. The range of integration in Eq. (20) should be such that the correct range of shower energy can be obtained. As we consider the $\tau$ lepton as the origin of observed events, with $E_{\tau}=E_{\nu} / 4$ and observed shower energy $0.2-1 \mathrm{EeV}$, we take $E_{i}=0.8 \mathrm{EeV}, E_{f}=4 \mathrm{EeV}$. As far as the flux $\Phi\left(E_{\nu}\right)$ is concerned, for the isotropic case, if one assumes the source of the $\mathrm{EeV}$ neutrinos to be the Greisen-Zatspin-Kuzmin (GZK) mechanism, then in that case, following Ref. [41], for the concerned shower energy, $\Phi_{\text {iso }} \approx 10^{-25}\left(\mathrm{GeV} \mathrm{cm}^{2} \mathrm{~s} \mathrm{sr}\right)^{-1}$. Otherwise, if some localized source is the origin of the $\mathrm{EeV}$ neutrinos, then the upper limit on such anisotropic flux would be $\Phi_{\text {aniso }} \approx$ $3.2 \times 10^{-20}\left(\mathrm{GeV} \mathrm{cm}^{2} \mathrm{~s} \mathrm{sr}\right)^{-1}$ [42-46]. Thus, by considering the mentioned parameters in the isotropic and anisotropic cases to get two AAEs, one should have

$$
\mathcal{N} \approx\left\{\begin{array}{l}
2.0 \times 10^{2} \epsilon \text { for isotropic case } \\
2.0 \times 10^{4} \epsilon \text { for anisotropic case }
\end{array}\right.
$$

This implies that for the observed two AAEs in the isotropic case, the $\epsilon$ should be $\sim 10^{-2}$, and for the anisotropic case $\epsilon$ should be $\sim 10^{-4}$. By considering SM interactions, the authors of Ref. [15] predicted the survival probability $\epsilon_{\mathrm{SM}} \sim 10^{-7}$, giving $\mathcal{N} \sim 2 \times 10^{-5}$ for the isotropic flux. Clearly in the SM, explanation of these two events is very unlikely. While using the SM interaction and considering the anisotropic flux, one gets $\mathcal{N} \sim 2 \times 10^{-3}$, which again makes it very implausible to get an explanation of the observed events in the SM. Thus, in any BSM scenario trying to explain the two AAEs, one should strive to increase the $\epsilon\left(E_{\nu}\right)$, and we will show that this can be achieved in one of the SM extensions that we are considering.

In the following section, we discuss the shower events, generated from $\tau$ decay, which is interpreted as the origin of the AAEs. Basically, $\tau$, which induces the air shower above the Antarctic surface, could also pass through the IceCube detector giving similar neutrino events, but no such event is observed by the IceCube Collaboration. In Ref. [15], the authors have interpreted three events of $\mathrm{PeV}$ energies in IceCube data [41] as sub-EeV Earth-emergent cosmic rays similar to ANITA. The exposure of IceCube is estimated at $54.0 \mathrm{~km}^{2} \mathrm{sryr}$, which is 20 times that of the ANITA exposure, $2.7 \mathrm{~km}^{2} \mathrm{sryr}$ [15]. By using the relative exposures, the number of events $\mathcal{N}$ at ANITA can be estimated as $\mathcal{N} \approx \mathcal{N}_{\mathcal{I C}} / 20$, giving $\mathcal{N}=0.15$ for $\mathcal{N}_{\mathcal{I C}}=3$ events at IceCube, which is roughly an order of magnitude away from the observed events by ANITA. As pointed out in Ref. [15], the ANITA estimated event rate serves as an upper limit which can be reduced for a better angular coverage, and a detailed analysis including particular instrumental effects is needed to resolve it. In the following section, while remaining consistent with IceCube, we will estimate the required survival probability for $\mathcal{N}=0.15$. In 
the estimation of survival probability, we have closely followed the approach of Ref. [19].

As discussed in the considered scenarios, the NLSP decays to LSP and a $\tau$ lepton, which is assumed to be the origin of the observed AAEs. While propagating through the Earth, the NLSP may lose its energy through electromagnetic processes. The average energy loss of a particle traveling a distance $l$ can be estimated from the equation $-d E / d l=\beta(E) E \rho(l)$, where $\rho(l)$ is the density of the Earth, and $\beta$ is the radiative energy loss. While using the parametrization for $\beta(E)$ from Ref. [47], we found $\beta=$ $6.5 \times 10^{-10} \mathrm{~cm}^{2} / \mathrm{gm}$ for NLSP initial energy $E=1 \mathrm{EeV}$ and mass $M_{\mathrm{NLSP}}=1 \mathrm{TeV}$. Then, with $\rho=4 \mathrm{gm} / \mathrm{cm}^{3}$ [48] for $l \sim 7000 \mathrm{~km}$, one can find the energy loss due to radiation to be $\sim 17 \%$, implying the NLSP energy to be $E \sim 0.8 \mathrm{EeV}$. The corresponding final shower energy $E / 2 \sim 0.4 \mathrm{EeV}$ is consistent with the ANITA observations.

\section{A. Model I}

The basic idea is to consider the ultrahigh-energy (UHE) neutrino interactions with the matter inside Earth. At the elementary level, the incoming UHE neutrino with energy $E_{\nu}$ interacts with the quarks mediated by the appropriate new physics particle and produces the BSM particle, which travels through the Earth and decays to a $\tau$ lepton, which actually gives rise to the AAEs. Given the particle content shown in Table I and the interactions in Eq. (1), the relevant processes in this case are $\nu \bar{d} \rightarrow \Phi_{2}^{-} \psi_{2}$ and $\nu \bar{u} \rightarrow \Phi_{2}^{-} \psi_{1}$, which are mediated by the particle $E$, where $\Phi_{2}^{-}$is the charged scalar arising from the doublet $\Phi_{2}$. In this case, the final $\tau$ lepton is obtained from the decay of the $\Phi_{2}^{-}$, which decays to $N_{1}$ and $\tau$. In this model, $N_{1}$ is identified as the DM candidate. In terms of Bjorken scaling variables $x=Q^{2} / 2 m_{N} E_{\nu}^{\prime}, \quad y=E_{\nu}^{\prime} / E_{\nu}$, where $m_{N}=\left(m_{p}+m_{n}\right) / 2$, the average mass of the proton and neutron, $E_{\nu}^{\prime}=E_{\nu}-E_{\Phi_{2}^{-}}$is the energy loss in the lab frame, and $-Q^{2}$ is the invariant momentum transfer between the neutrino (antineutrino) and particle $\Phi_{2}^{-}$, the differential cross-section of neutrino (antineutrino) nucleon scattering can be written as

$$
\begin{aligned}
\frac{d^{2} \sigma}{d x d y}= & \frac{Y_{2}^{2}}{32 \pi\left(Q^{2}+M_{E}^{2}\right)^{2}} \\
& \times x s y(1-y)\left(Y_{4}^{2}+Y_{5}^{2}\right)\left(f_{\bar{u}}\left(x, Q^{2}\right)+f_{\bar{d}}\left(x, Q^{2}\right)\right),
\end{aligned}
$$

where $s=2 m_{N} E_{\nu}$ is the center-of-mass energy, and $f_{q}\left(x, Q^{2}\right)$ and $f_{\bar{q}}\left(x, Q^{2}\right)$ are the parton distribution functions (PDFs) of quarks and antiquarks with $q=u, d$. In our analysis, we consider the standard $(1: 1: 1)$ flavor ratio of the neutrino (antineutrino) on Earth and use CTEQ6 leading-order PDF sets [49] while calculating the cross sections. By using the interaction cross section obtained from Eq. (23), the interaction length, $l_{\Phi_{2}}$, can be written as

$$
l_{\Phi_{2}^{-}}=\frac{1}{\rho \sigma N_{A}},
$$

where $N_{A}=6.022 \times 10^{23} \mathrm{~cm}^{-3}$ is the Avogadro number and $\rho \approx 4$ is the Earth's density in water-equivalent units. A convenient representation of the density profile of the Earth is given in Ref. [48], which is used to calculate the approximate value of $\rho$ for the relevant chord lengths for the AAEs. The decay length of $\Phi_{2}^{-}, l_{D}$, in the Earth rest frame can be written as

$$
l_{D}=\gamma c \tau=\frac{1}{\Gamma_{\Phi_{2}^{-}}} \frac{E_{\Phi_{2}^{-}}}{M_{\Phi_{2}^{-}}}
$$

where $\Gamma_{\Phi_{2}^{-}} \equiv \Gamma\left(\Phi_{2}^{-} \rightarrow \tau^{-} N_{1}\right)$ is the rest-frame decay width of $\Phi_{2}^{-}$into $\tau$ leptons and DM $N_{1}$, which is obtained from Eq. (7). Here we use the approximation $E_{\Phi_{2}^{-}}=$ $(1-\xi) E_{\nu} / 2$, where $\xi$ accounts for the energy loss of $\Phi_{2}^{-}$ due to radiation (which is $\sim 17 \%$, as estimated before), to get the observed shower energy $E_{\tau}=E_{\Phi_{2}^{-}} / 2 \sim 0.4 \mathrm{EeV}$ by taking into account the incident neutrino energy $E_{\nu}=2 \mathrm{EeV}$. As mentioned before, by considering only the SM interactions, it is not possible to get the desired survival probability for any SM particle to pass a chord distance of $\sim 7000 \mathrm{~km}$. In considered BSM scenarios, as a result of additional interactions, the survival probability of the neutrino flux can be estimated as [19]

$$
\begin{aligned}
\epsilon_{\mathrm{BSM}}= & \int_{0}^{l_{\oplus}} d l_{1} \int_{l_{\oplus}-l_{1}-h}^{l_{\oplus}-l_{1}} d l_{2} \frac{e^{-l_{2} / l_{D}}}{l_{D}} \frac{e^{-l_{1} / l_{\Phi_{2}^{-}}}}{l_{\Phi_{2}^{-}}} \\
& \times\left(1-\int_{0}^{l_{1}} \frac{d l_{3}}{l_{0}} e^{-l_{3} / l_{0}}\right),
\end{aligned}
$$

where $l_{\oplus}$ is the chord length; $l_{0}$ is the mean interaction length, which is 290 and $265 \mathrm{~km}$ for ANITA-I and ANITAIII events, respectively [15]; and $h \approx 10 \mathrm{~km}$ is the window of $\tau$ production near the surface of Earth.

In Fig. 4, we plot the survival probability $\epsilon_{\mathrm{BSM}}$ of $\Phi_{2}^{-}$by normalizing it with respect to $\epsilon_{\mathrm{SM}}$, as a function of $M_{\Phi_{2}^{-}}$, and its decay coupling $Y_{3}$ with DM and tau leptons, for ANITA-I (left) and ANITA-III (right) events. While scanning the parameter space, we considered the benchmark relevant for the correct relic density, which are quoted in Table II and shown as a star in Fig. 4 for $M_{\Phi_{2}^{-}}=1 \mathrm{TeV}$ and $Y_{3}=2.92 \times 10^{-10}$. The color scheme corresponds to different regions of parameter space where $\epsilon_{\mathrm{BSM}} / \epsilon_{\mathrm{SM}}^{I, I I I} \geq 1$. In our analysis, we considered $\epsilon_{\mathrm{SM}}^{I}=4.4 \times 10^{-7}$ and $\epsilon_{\mathrm{SM}}^{I I I}=$ $3.2 \times 10^{-8}$ for ANITA-I and ANITA-III events, respectively [15]. By using the benchmark in this model, we get $\epsilon_{\mathrm{BSM}}=2.45 \times 10^{-7}$ for ANITA-I events and $\epsilon_{\mathrm{BSM}}=$ $2.17 \times 10^{-7}$ for ANITA-III events. When these values are used in Eq. (22), we find $\mathcal{N}=0.00009$ for isotropic 

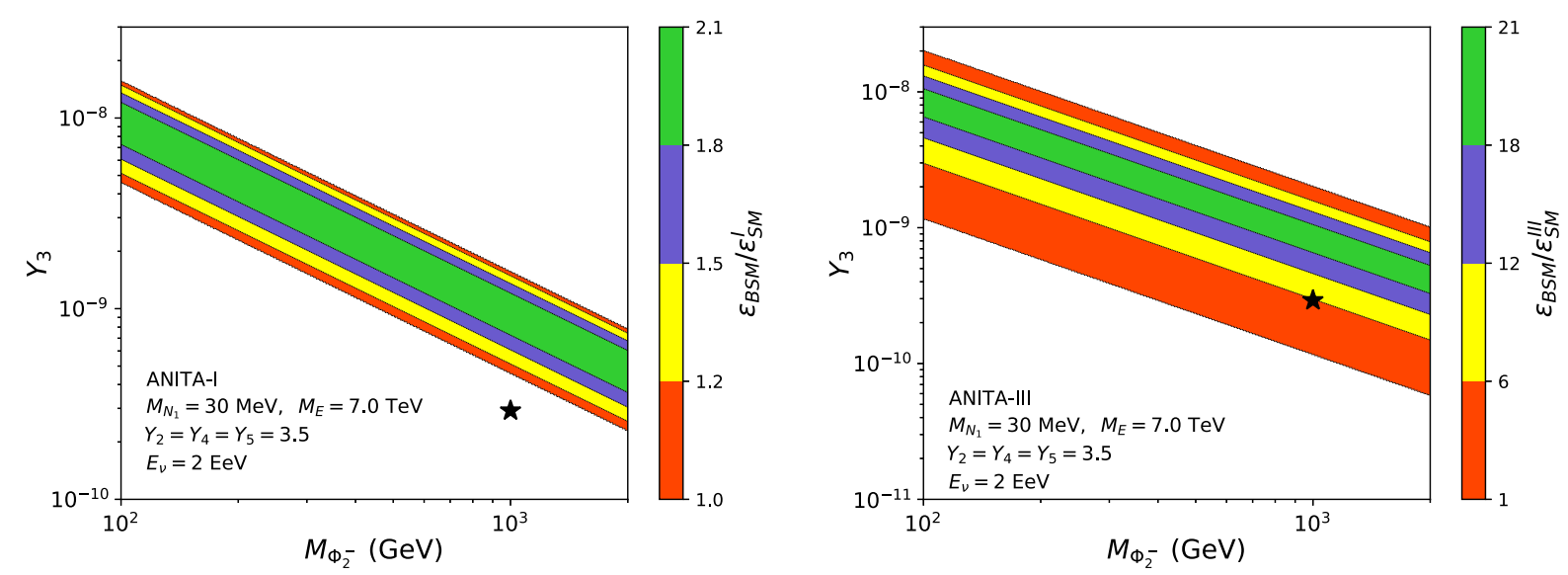

FIG. 4. Model I: The survival probability $\epsilon_{\mathrm{BSM}}$ of $\Phi_{2}^{-}$normalized to $\epsilon_{\mathrm{SM}}^{I}$ (left) and $\epsilon_{\mathrm{SM}}^{I I I}$ (right) as a function of $M_{\Phi_{2}^{-}}$and its decay coupling $Y_{3}$ to DM and tau leptons. Here $\epsilon_{\mathrm{SM}}^{I}$ and $\epsilon_{\mathrm{SM}}^{I I I}$ are SM survival probabilities for ANITA-I and ANITA-III events, respectively. The benchmark consistent with the relic density calculation is shown as a star.

flux and $\mathcal{N}=0.009$ for anisotropic flux. Clearly, in this model, it is not possible to get the desired number of events for perturbative Yukawa couplings while being consistent with DM relic density estimation.

\section{B. Model II}

The basic tenet of explaining the AAEs in this model follows a similar path as Model I, mentioned in the previous subsection. In Model II, outlined in Sec. II A, the necessary interactions can be obtained from the Yukawa Lagrangian defined in Eq. (10). In this case, the typical relevant processes would be $\nu d \rightarrow E U$ and $\nu \bar{u} \rightarrow E \bar{D}$, mediated by the charged scalar arising from the doublet $\Phi_{2}$. The produced $E$ subsequently decays to $\tau$ and the DM candidate, which is the neutral component of $\Phi_{3}$. Now, for the mentioned processes, the total differential cross section of neutrino (antineutrino) scattering in terms of Bjorken scaling variables $x=Q^{2} / 2 m_{N} E_{\nu}^{\prime}, y=E_{\nu}^{\prime} / E_{\nu}$, with $E_{\nu}^{\prime}=$ $E_{\nu}-E_{E}$ being the energy loss in the lab frame, and $-Q^{2}$ the invariant momentum transfer between the neutrino (antineutrino) and particle $E$, can be written as

$$
\begin{aligned}
\frac{d^{2} \sigma}{d x d y}= & \frac{x s y^{2} Y_{2}^{2}}{16 \pi\left(Q^{2}+M_{\Phi_{2}^{ \pm}}^{2}\right)^{2}}\left[Y _ { 4 } ^ { 2 } \left(f_{u}\left(x, Q^{2}\right)\right.\right. \\
& \left.\left.+f_{\bar{u}}\left(x, Q^{2}\right)\right)+Y_{5}^{2}\left(f_{d}\left(x, Q^{2}\right)+f_{\bar{d}}\left(x, Q^{2}\right)\right)\right],
\end{aligned}
$$

where $s=2 m_{N} E_{\nu}$ is the center-of-mass energy. Similarly to the previous case, the interaction length, $l_{E}$, can be given as

$$
l_{E}=\frac{1}{\rho \sigma N_{A}},
$$

where $\sigma$ is the interaction cross section obtained from Eq. (27). The decay length of $E$ in the Earth rest frame can be written as

$$
l_{D}=\gamma c \tau=\frac{1}{\Gamma_{E}} \frac{E_{E}}{M_{E}}
$$

where $\Gamma_{E} \equiv \Gamma\left(E \rightarrow \tau^{-} \Phi_{3}^{0}\right)$ is the rest-frame decay width of $E$ obtained from Eq. (18). As before, we use the approximation $E_{E}=(1-\xi) E_{\nu} / 2$, where $\xi$ accounts for particle $E$ energy loss due to radiation (which is $\sim 17 \%$, as estimated before), to get the observed shower energy $E_{\tau}=E_{E} / 2 \sim$ $0.4 \mathrm{EeV}$ by taking into account the incident neutrino energy $E_{\nu}=2 \mathrm{EeV}$. In this case, the survival probability of the neutrino flux, $\epsilon_{\mathrm{BSM}}$, can also be written following Eq. (26) by making the substitution $l_{\Phi_{2}^{-}} \rightarrow l_{E}$ and the appropriate $l_{D}$ defined in Eq. (29).

In Fig. 5, we plot the survival probability $\epsilon_{\mathrm{BSM}}$ of $E$ normalized to $\epsilon_{\mathrm{SM}}$, as a function of its mass $M_{E}$ and its decay coupling $Y_{2}^{\prime}$ to DM and tau leptons for ANITA-I (left) and ANITA-III (right) events. While scanning the parameter space, we considered the parameters relevant for correct relic density, which are quoted in Table IV, and specifically the first benchmark point is shown as a star in Fig. 5 for $M_{E}=1 \mathrm{TeV}$ and $Y_{2}^{\prime}=1.1 \times 10^{-9}$. The color scheme corresponds to different regions of parameter space where $\epsilon_{\mathrm{BSM}} / \epsilon_{\mathrm{SM}}^{I, I I I} \geq 1$. While using the first benchmark point, we get $\epsilon_{\mathrm{BSM}}=4.33 \times 10^{-6}$ for ANITAI events and $\epsilon_{\mathrm{BSM}}=3.41 \times 10^{-6}$ for ANITA-III events. These values are then used in Eq. (22), and we find $\mathcal{N}=$ 0.0015 for isotropic flux, and $\mathcal{N}=0.154$ for anisotropic flux. We repeat a similar exercise for the second benchmark point by taking into account the relic density estimations and get $\mathcal{N}=0.17$ for anisotropic flux. Our result for the second benchmark point is shown in Fig. 6 . Clearly for both the benchmarks, it is not possible to get the desired survival probability for isotropic flux, and so anisotropic flux is required while being consistent with DM relic density estimation. 

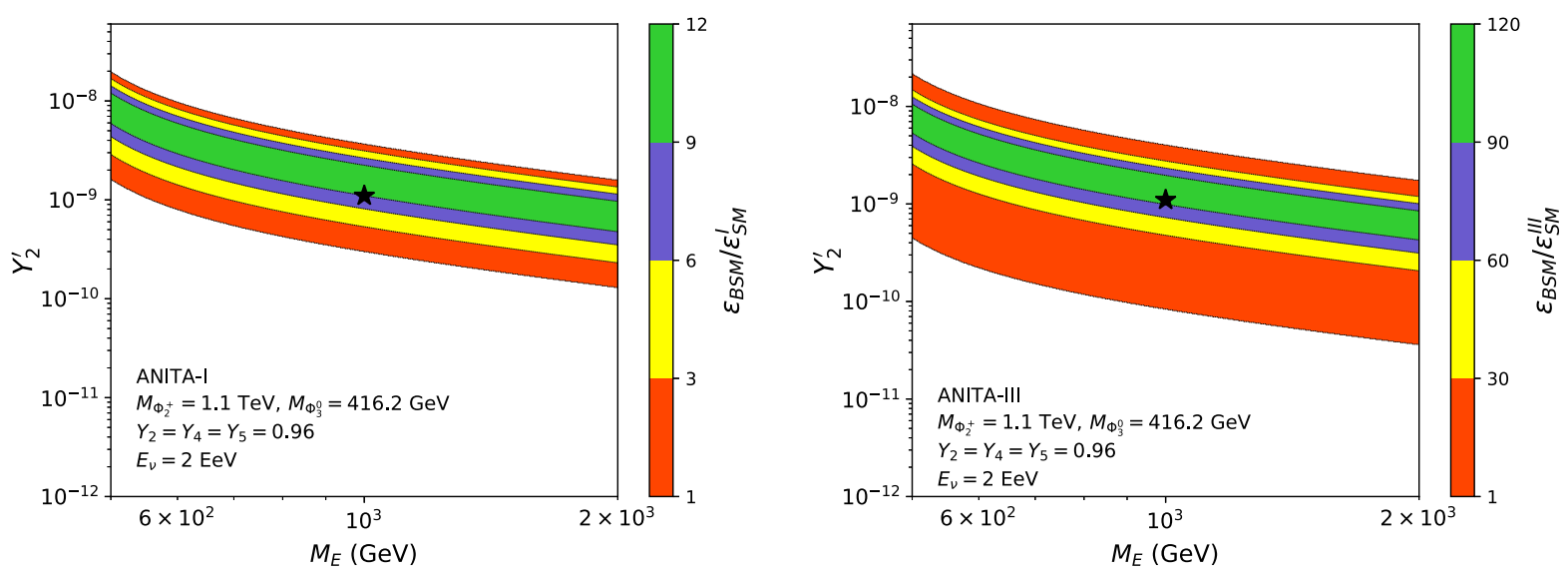

FIG. 5. Model II: The survival probability $\epsilon_{\mathrm{BSM}}$ of $E$ normalized to $\epsilon_{\mathrm{SM}}^{I}$ (left) and $\epsilon_{\mathrm{SM}}^{I I I}$ (right) as a function of fermion mass $M_{E}$ and its decay coupling $Y_{2}^{\prime}$ to DM and tau leptons. Here $\epsilon_{\mathrm{SM}}^{I}$ and $\epsilon_{\mathrm{SM}}^{I I I}$ are SM survival probabilities for ANITA-I and ANITA-III events, respectively. The benchmark consistent with the relic density calculation is shown as a star.
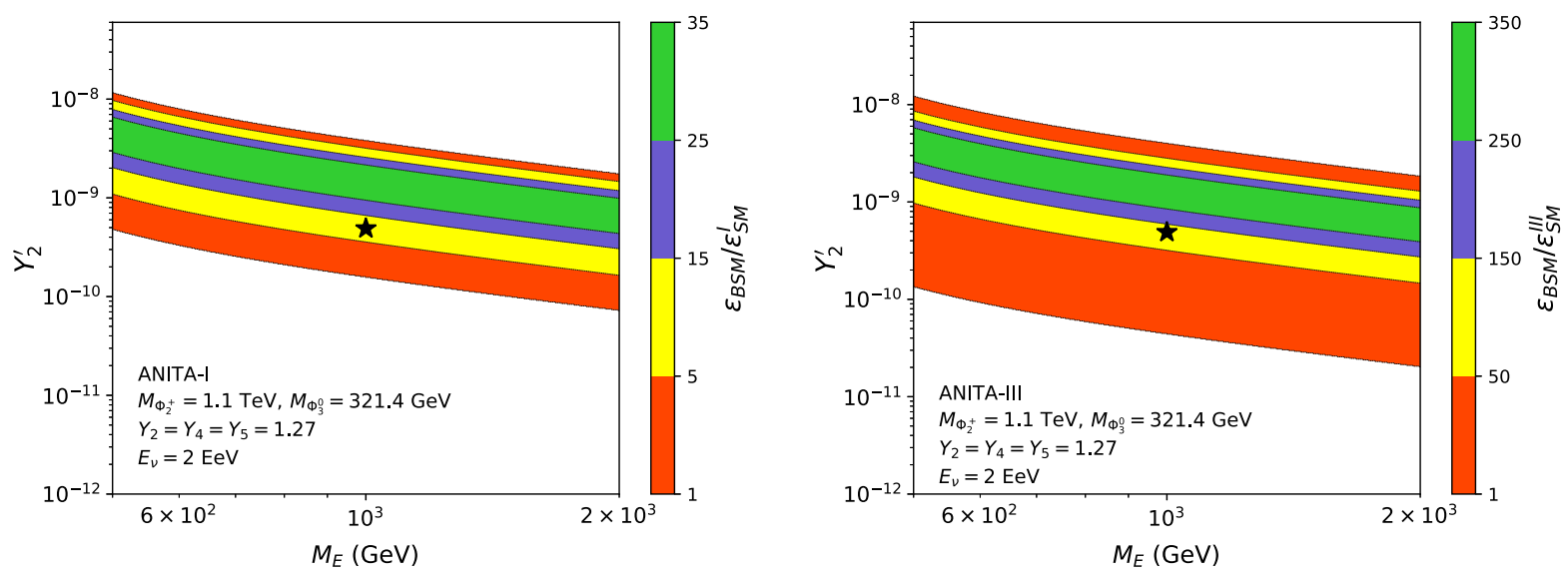

FIG. 6. Same as Fig. 5 for the second benchmark.

\section{COMPLEMENTARY PROBES OF THE MODEL}

Apart from providing an explanation to AAEs along with dark matter, the model we propose here can also offer tantalizing signatures at different experiments operating at energy as well as intensity frontiers. We briefly comment on such possibilities related to Model II, which is successful in explaining the observed AAEs discussed before. Clearly, the model is an extension of the widely studied inert Higgs doublet model or scotogenic model of radiative neutrino mass with additional $Z_{2}$-odd particles. The scalar sector of Model II contains two $Z_{2}$-odd scalar doublets $\Phi_{2,3}$. By virtue of their electroweak gauge interactions, the components of these scalar doublets can be produced significantly in proton-proton collisions of the Large Hadron Collider (LHC). Depending upon the mass spectrum of its components, the heavier ones can decay into the lighter ones and a gauge boson, which finally decays into a pair of leptons or quarks. Therefore, we can have either pure leptonic final states plus missing transverse energy
(MET), hadronic final states plus MET, or a mixture of both. The MET corresponds to DM or light neutrinos. In several earlier works [50-52], the possibility of oppositesign dileptons plus MET was discussed. In Ref. [53], the possibility of dijet plus MET was investigated with the finding that inert scalar masses up to $400 \mathrm{GeV}$ can be probed at high-luminosity LHC. In another work [54], trilepton plus MET final states were also discussed, whereas monojet signatures have been studied by the authors of Refs. [55,56]. The enhancement in the dilepton plus MET signal in the presence of additional vectorlike singlet charged leptons (like we have in Model II) was also discussed in Ref. [57]. Exotic signatures like the displaced vertex and the disappearing or long-lived charged track for a compressed mass spectrum of inert scalars and singlet fermion DM were studied recently by the authors of Ref. [58].

To summarize, the search strategy and the bounds on $Z_{2}$-odd scalars depend crucially on the model and spectrum of lighter particles to which they can decay. Due to identical 
gauge interactions, the bounds are somewhat similar to the ones on sleptons in supersymmetric models. As mentioned in Ref. [33], collider bounds on charged sleptons are also dependent upon the lightest neutralino mass among other details related to the mass spectrum. Since we are not performing a detailed collider analysis in our work, we consider a conservative lower bound on such $Z_{2}$-odd charged scalar masses $(>100 \mathrm{GeV})$ in agreement with LEP bounds $[33,59]$. Also, the presence of $Z_{2}$-odd vectorlike singlet charged leptons can lead to dilepton plus MET signatures at colliders. As noted from the collider studies in Refs. $[57,60]$ within a similar model, the chosen values of vectorlike charged lepton masses are safe from existing collider limits. As can be seen from the above analysis, such bounds are satisfied by the parameter space shown in Figs. 4, 5, and 6.

Apart from collider signatures of inert scalars in the model, as discussed in several earlier works mentioned above, the model can also have interesting signatures at lepton-flavor-violating decays like $\mu \rightarrow e \gamma, \mu \rightarrow e e e$, and $\mu \rightarrow e$ conversions [61,62]. With the inclusion of vectorlike quarks and vectorlike charged leptons, like we have in Model II, one can also explain flavor anomalies in $b \rightarrow s$ decays as well as muon $g-2$ [60]. Thus, the model we have studied here, which successfully explains the ANITA anomalous events, also has rich phenomenology that can be accessed at different experiments operating at cosmic energy as well as intensity frontiers.

\section{SUMMARY AND CONCLUSIONS}

We have proposed a beyond-standard-model framework to explain the two anomalous upward-going ultrahighenergy cosmic-ray air shower events reported by the ANITA Collaboration. The novel feature of the model is the way it relates the origin of AAEs to the nonthermal origin of dark matter in the Universe as well as the origin of light neutrino masses. Sticking to minimality, we extend the standard model by a few additional particles, all of which are odd under an in-built and unbroken $Z_{2}$ symmetry. While two types of such $Z_{2}$-odd fields-namely, a scalar doublet and gauge singlet neutral fermions-play the role of generating light neutrino masses at the one-loop level similar to the scotogenic scenarios, the other fields are responsible for generating the AAEs. The nonthermal nature of dark matter is related to its tiny coupling with the next-to-lightest $Z_{2}$-odd particle, whose long-livedness makes its passage through the Earth possible, followed by its decay into DM and a tau lepton required to explain the AAEs.

We first consider a dark matter scenario where its relic is generated purely from nonthermal production. In this case, one of the three $Z_{2}$-odd gauge singlet neutral fermions is the DM candidate. Being gauge singlet, its nonthermal nature is dictated by its tiny coupling with the SM leptons and $Z_{2}$-odd scalar doublet. While such tiny coupling results in a vanishingly small lightest neutrino mass [37], the abundance of DM is generated from decay of the $Z_{2}$-odd scalar doublet, the NLSP in this case. While the correct DM relic is obtained by suitable choices of DM, NLSP masses, and their coupling, it was found that the model cannot explain the AAEs. We then consider a hybrid setup, where the DM relic is obtained from thermal as well as nonthermal contributions. The neutral component of a second $Z_{2}$-odd scalar doublet is considered to be the DM candidate which annihilates into the SM particles at a rate larger than the one required for correct thermal relic abundance. This leads to an underabundant thermal dark matter, and the deficit can be filled in by a nonthermal contribution from a singlet fermion, considered to be a charged vectorlike singlet fermion $E$. The light neutrino masses arise as usual from gauge singlet neutral fermions and another $Z_{2}$-odd scalar doublet, thereby not getting affected by the smallness of the DM coupling with $E$. The model is found to explain the AAEs successfully.

Although we have confined our discussions here to DM relics and anomalous ANITA events, Model II of our study, successful in explaining both of these, can have other consequences which can be tested at experiments operating at different frontiers. For example, the components of $Z_{2}$ odd scalar doublets can be produced at a significant rate at collider experiments like the LHC due to its electroweak gauge interactions. Our model also has additional colored vectorlike $Z_{2}$-odd fermions, which can also give rise to similar signatures at colliders. While we briefly mention such additional aspects of probing this model at a variety of experiments, the detailed phenomenological study of such scenarios is beyond the scope of this present work and left for future works.

\section{ACKNOWLEDGMENTS}

D. B. acknowledges the support from IIT Guwahati start-up Grant (Reference No. xPHYSUGIITG01152xxDB001), the Early Career Research Award from DST-SERB, the Government of India (Reference No. ECR/2017/001873) and the Associateship Programme of Inter University Centre for Astronomy and Astrophysics (IUCAA), Pune. The research of U.K. D. is supported by the Ministry of Science, ICT \& Future Planning of Korea, the Pohang City Government, and the Gyeongsangbuk-do Provincial Government. The research of G. T. is supported by the Basic Science Research Program through the National Research Foundation of Korea (NRF) funded by the Ministry of Education, Grant No. 2019R1F1A1052231. D. B. and U. K. D. thank the organizers of WHEPP XVI, where important discussions were carried out. We would like to thank Bhavesh Chauhan for useful discussion. 
[1] P. W. Gorham et al., Characteristics of Four UpwardPointing Cosmic-Ray-Like Events Observed with ANITA, Phys. Rev. Lett. 117, 071101 (2016).

[2] P. W. Gorham et al., Observation of an Unusual UpwardGoing Cosmic-Ray-Like Event in the Third Flight of ANITA, Phys. Rev. Lett. 121, 161102 (2018).

[3] J. F. Cherry and I. M. Shoemaker, Sterile neutrino origin for the upward directed cosmic ray showers detected by ANITA, Phys. Rev. D 99, 063016 (2019).

[4] G.-Y. Huang, Sterile neutrinos as a possible explanation for the upward air shower events at ANITA, Phys. Rev. D 98, 043019 (2018).

[5] L. A. Anchordoqui, V. Barger, J. G. Learned, D. Marfatia, and T. J. Weiler, Upgoing ANITA events as evidence of the CPT symmetric universe, Lett. High Energy Phys. 1, 13 (2018).

[6] E. Dudas, T. Gherghetta, K. Kaneta, Y. Mambrini, and K. A. Olive, Gravitino decay in high scale supersymmetry with R-parity violation, Phys. Rev. D 98, 015030 (2018).

[7] L. Heurtier, Y. Mambrini, and M. Pierre, A dark matter interpretation of the ANITA anomalous events, Phys. Rev. D 99, 095014 (2019).

[8] D. Hooper, S. Wegsman, C. Deaconu, and A. Vieregg, Superheavy dark matter and ANITA's anomalous events, Phys. Rev. D 100, 043019 (2019).

[9] S. Chipman, R. Diesing, M. H. Reno, and I. Sarcevic, Anomalous ANITA air shower events and tau decays, Phys. Rev. D 100, 063011 (2019).

[10] L. Heurtier and F. Huang, The inflaton portal to a highly decoupled EeV dark matter particle, Phys. Rev. D 100, 043507 (2019).

[11] W. Yin, Highly-boosted dark matter and cutoff for cosmicray neutrinos through neutrino portal, EPJ Web Conf. 208, 04003 (2019).

[12] W. Yin, Dark matter, neutrino mass, cutoff for cosmic-ray neutrino, and the Higgs boson invisible decay from a neutrino portal interaction, Chin. Phys. C 43, 045101 (2019).

[13] J. M. Cline, C. Gross, and W. Xue, Can the ANITA anomalous events be due to new physics?, Phys. Rev. D 100, 015031 (2019).

[14] L. Heurtier, D. Kim, J.-C. Park, and S. Shin, Explaining the ANITA anomaly with inelastic boosted dark matter, Phys. Rev. D 100, 055004 (2019).

[15] D. B. Fox, S. Sigurdsson, S. Shandera, P. Mészáros, K. Murase, M. Mostafá, and S. Coutu, The ANITA anomalous events as signatures of a beyond standard model particle, and supporting observations from IceCube, arXiv: 1809.09615.

[16] A. Connolly, P. Allison, and O. Banerjee, On ANITA's sensitivity to long-lived, charged massive particles, arXiv:1807.08892.

[17] J.H. Collins, P.S. Bhupal Dev, and Y. Sui, R-parity violating supersymmetric explanation of the anomalous events at ANITA, Phys. Rev. D 99, 043009 (2019).

[18] L. A. Anchordoqui and I. Antoniadis, Supersymmetric sphaleron configurations as the origin of the perplexing ANITA events, Phys. Lett. B 790, 578 (2019).
[19] B. Chauhan and S. Mohanty, Leptoquark solution for both the flavor and ANITA anomalies, Phys. Rev. D 99, 095018 (2019).

[20] I. Esteban, J. Lopez-Pavon, I. Martinez-Soler, and J. Salvado, Looking at the axionic dark sector with ANITA, arXiv:1905.10372.

[21] A. Romero-Wolf et al., Comprehensive analysis of anomalous ANITA events disfavors a diffuse tau-neutrino flux origin, Phys. Rev. D 99, 063011 (2019).

[22] A. Aab et al., Improved limit to the diffuse flux of ultrahigh energy neutrinos from the Pierre Auger Observatory, Phys. Rev. D 91, 092008 (2015).

[23] M. G. Aartsen et al., Constraints on Ultrahigh-Energy Cosmic-Ray Sources from a Search for Neutrinos above $10 \mathrm{PeV}$ with IceCube, Phys. Rev. Lett. 117, 241101 (2016); Erratum, Phys. Rev. Lett. 119, 259902 (2017).

[24] K. D. de Vries and S. Prohira, Coherent Transition Radiation from the Geomagnetically-Induced Current in Cosmic-Ray Air Showers: Implications for the Anomalous Events Observed by ANITA, Phys. Rev. Lett. 123, 091102 (2019).

[25] I. M. Shoemaker, A. Kusenko, P. K. Munneke, A. RomeroWolf, D. M. Schroeder, and M. J. Siegert, Reflections On the anomalous ANITA events: The antarctic subsurface as a possible explanation, arXiv:1905.02846.

[26] N. Aghanim et al., Planck 2018 results. VI. Cosmological parameters, arXiv:1807.06209.

[27] L. J. Hall, K. Jedamzik, J. March-Russell, and S. M. West, Freeze-in production of FIMP dark matter, J. High Energy Phys. 03 (2010) 080.

[28] J. König, A. Merle, and M. Totzauer, keV sterile neutrino dark matter from singlet scalar decays: The most general case, J. Cosmol. Astropart. Phys. 11 (2016) 038.

[29] A. Biswas and A. Gupta, Freeze-in production of sterile neutrino dark matter in $\mathrm{U}(1)_{\mathrm{B}-\mathrm{L}}$ Model, J. Cosmol. Astropart. Phys. 09 (2016) 044; 05 (2017) A01.

[30] A. Biswas and A. Gupta, Calculation of momentum distribution function of a non-thermal fermionic dark matter, J. Cosmol. Astropart. Phys. 03 (2017) 033; 05 (2017) A02.

[31] N. Bernal, M. Heikinheimo, T. Tenkanen, K. Tuominen, and V. Vaskonen, The dawn of FIMP dark matter: A review of models and constraints, Int. J. Mod. Phys. A 32, 1730023 (2017).

[32] E. Ma, Verifiable radiative seesaw mechanism of neutrino mass and dark matter, Phys. Rev. D 73, 077301 (2006).

[33] M. Tanabashi et al., Review of particle physics, Phys. Rev. D 98, 030001 (2018).

[34] P. Minkowski, $\mu \rightarrow e \gamma$ at a rate of one out of $10^{9}$ muon decays? Phys. Lett. B 67, 421 (1977).

[35] R. N. Mohapatra and G. Senjanovic, Neutrino Mass and Spontaneous Parity Violation, Phys. Rev. Lett. 44, 912 (1980).

[36] J. Schechter and J. W. F. Valle, Neutrino masses in $S U(2) \times$ $U(1)$ theories, Phys. Rev. D 22, 2227 (1980).

[37] D. Borah and A. Gupta, New viable region of an inert Higgs doublet dark matter model with scotogenic extension, Phys. Rev. D 96, 115012 (2017). 
[38] A. Biswas, D. Borah, and D. Nanda, When freeze-out precedes freeze-in: Sub-TeV fermion triplet dark matter with radiative neutrino mass, J. Cosmol. Astropart. Phys. 09 (2018) 014.

[39] J. L. Feng, A. Rajaraman, and F. Takayama, SuperWIMP dark matter signals from the early Universe, Phys. Rev. D 68, 063504 (2003).

[40] K. Griest and D. Seckel, Three exceptions in the calculation of relic abundances, Phys. Rev. D 43, 3191 (1991).

[41] M. G. Aartsen et al., Differential limit on the extremelyhigh-energy cosmic neutrino flux in the presence of astrophysical background from nine years of IceCube data, Phys. Rev. D 98, 062003 (2018).

[42] I. B. Jacobsen, K. Wu, A. Y. L. On, and C. J. Saxton, Highenergy neutrino fluxes from AGN populations inferred from X-ray surveys, Mon. Not. R. Astron. Soc. 451, 3649 (2015).

[43] S. Adrian-Martinez et al., The first combined search for neutrino point-sources in the southern hemisphere with the antares and Icecube neutrino telescopes, Astrophys. J. 823, 65 (2016).

[44] M. G. Aartsen et al., All-sky search for time-integrated neutrino emission from astrophysical sources with $7 \mathrm{yr}$ of IceCube data, Astrophys. J. 835, 151 (2017).

[45] P. Mertsch, M. Rameez, and I. Tamborra, Detection prospects for high energy neutrino sources from the anisotropic matter distribution in the local universe, J. Cosmol. Astropart. Phys. 03 (2017) 011.

[46] T. Fujii, Future ground arrays for ultrahigh-energy cosmic rays: Recent updates and perspectives, EPJ Web Conf. 208, 15004 (2019).

[47] M. H. Reno, I. Sarcevic, and S. Su, Propagation of supersymmetric charged sleptons at high energies, Astropart. Phys. 24, 107 (2005).

[48] R. Gandhi, C. Quigg, M. H. Reno, and I. Sarcevic, Ultrahigh-energy neutrino interactions, Astropart. Phys. 5, 81 (1996).

[49] J. Pumplin, D. R. Stump, J. Huston, H. L. Lai, P. M. Nadolsky, and W. K. Tung, New generation of parton distributions with uncertainties from global QCD analysis, J. High Energy Phys. 07 (2002) 012.
[50] X. Miao, S. Su, and B. Thomas, Trilepton signals in the inert doublet model, Phys. Rev. D 82, 035009 (2010).

[51] M. Gustafsson, S. Rydbeck, L. Lopez-Honorez, and E. Lundstrom, Status of the inert doublet model and the role of multileptons at the LHC, Phys. Rev. D 86, 075019 (2012).

[52] A. Datta, N. Ganguly, N. Khan, and S. Rakshit, Exploring collider signatures of the inert Higgs doublet model, Phys. Rev. D 95, 015017 (2017).

[53] P. Poulose, S. Sahoo, and K. Sridhar, Exploring the inert doublet model through the dijet plus missing transverse energy channel at the LHC, Phys. Lett. B 765, 300 (2017).

[54] M. Hashemi and S. Najjari, Observability of inert scalars at the LHC, Eur. Phys. J. C 77, 592 (2017).

[55] A. Belyaev, G. Cacciapaglia, I. P. Ivanov, F. Rojas, and M. Thomas, Anatomy of the inert two Higgs doublet model in the light of the LHC and non-LHC dark matter searches, Phys. Rev. D 97, 035011 (2018).

[56] A. Belyaev, T. R. Fernandez Perez Tomei, P. G. Mercadante, C. S. Moon, S. Moretti, S. F. Novaes, L. Panizzi, F. Rojas, and M. Thomas, Advancing LHC probes of dark matter from the inert two-Higgs-doublet model with the monojet signal, Phys. Rev. D 99, 015011 (2019).

[57] D. Borah, S. Sadhukhan, and S. Sahoo, Lepton portal limit of inert Higgs doublet dark matter with radiative neutrino mass, arXiv:1703.08674.

[58] D. Borah, D. Nanda, N. Narendra, and N. Sahu, Righthanded neutrino dark matter with radiative neutrino mass in gauged $B-L$ model, arXiv:1810.12920.

[59] E. Lundstrom, M. Gustafsson, and J. Edsjo, The inert doublet model and LEP II limits, Phys. Rev. D 79, 035013 (2009).

[60] B. Barman, D. Borah, L. Mukherjee, and S. Nandi, Correlating the anomalous results in $b \rightarrow s$ decays with inert Higgs doublet dark matter and muon $(g-2)$, Phys. Rev. D 100, 115010 (2019).

[61] T. Toma and A. Vicente, Lepton flavor violation in the scotogenic model, J. High Energy Phys. 01 (2014) 160.

[62] A. Vicente and C. E. Yaguna, Probing the scotogenic model with lepton flavor violating processes, J. High Energy Phys. 02 (2015) 144. 